\title{
BIENESTAR EN REGIONES EXTREMAS DE CHILE: COMPARACIÓN DE LA SATISFACCIÓN VITAL ENTRE MAGALLANES, ARICA Y PARINACOTA Y EL RESTO DEL PAÍS
}

SALVADOR VARGAS SALFATE', ${ }^{\mathrm{a}, \mathrm{f}}$ DARÍO PÁEZ ${ }^{\mathrm{b}, e}$, JUAN CARLOS OYANEDEL ${ }^{\mathrm{a}, \mathrm{c}} \&$ CAMILA MELLA ${ }^{\mathrm{d}}$

\section{RESUMEN}

Este estudio compara el bienestar subjetivo en Magallanes, Arica y Parinacota con el resto de las regiones de Chile utilizando los datos de la encuesta CASEN de 2011 sobre quintiles de ingreso, edad, sexo y un ítem de satisfacción con la vida. Los resultados evidencian que el bienestar subjetivo era mayor en Magallanes que en Arica y en el resto del país, aun controlando la edad, el sexo y los niveles de ingreso. Las diferencias en bienestar debidas a sexo y niveles de ingreso son menores en la Región de Magallanes, sugiriendo que la fuerte identidad colectiva y clima emocional positivo de esta región amortigua el impacto de las asimetrías de status y económicas. Este estudio aporta evidencia indirecta respecto a la existencia de una identidad social o colectiva regional magallánica positiva, que actúa como un factor de bienestar.

PALABRAS CLAVE: bienestar, regiones, edad, género, ingresos, identidad colectiva.

\section{WELL-BEING AT THE EXTREME REGIONS OF CHILE: A COMPARATION OF LIFE SATISFACTION LEVELS BETWEEN MAGALLANES, ARICA-PARINACOTA AND THE REST OF THE COUNTRY}

\footnotetext{
ABSTRACT

This paper compares subjective well-being in the regions of Magallanes and Arica-Parinacota with the rest of the regions. It makes use of the National Socio-Economic Characterisation Survey (CASEN), which includes information about quintiles income, age, sex and overall life satisfaction. Results show

Fundación Centro de Estudios Cuantitativos. Dirección: Bandera 84, oficina 112, Santiago, Chile. salvador.vargas@fcec.cl Universidad del País Vasco. Dirección: Av. De Tolosa 2019, San Sebastián, España. dario.paez@ehu.es

c Universidad Andrés Bello. Facultad de Educación. Dirección: Fernández Concha 700, Las Condes, Santiago, Chile. juan. oyanedel@unab.cl

d Department of Social Policy and Intervention, University of Oxford. Dirección: Barnett House, 32 -37 Wellington Square, Oxford OX12ER, UK. camila.mella@wolfson.ox.ac.uk

e Facultad de Administración y Economía, Universidad de Santiago. Av. Libertador Bernardo O`Higgins 3363, Santiago, Región Metropolitana

f Universidad de Girona. Dirección: Plaça Sant Domènec, 917071, Girona, España.
} 
that subjective well-being was higher in Magallanes than Arica and higher than the rest of country, even after controlling by age, sex and income. Differences in well-being due to sex and income are lower in Magallanes, suggesting that a strong collective identity and positive emotional climate from this region lessens the impact of status and income asymmetries. This study contributes indirect evidence about the existence of a positive social or collective regional identity in Magallanes, which acts a protective factor for well-being.

KEY WORDS: well-being, regions, age, gender, income, collective identity.

\section{INTRODUCCIÓN}

El bienestar subjetivo corresponde a las evaluaciones que desarrollan los propios individuos sobre sus vidas (Anger, 2010; Diener, 2009a). Una de las principales distinciones del bienestar se estructura en torno a los conceptos de bienestar eudaimónico y hedónico. De acuerdo a la primera taxonomía teórica, el bienestar refiere a la vivencia plena, realizando las diferentes potencialidades del ser humano, buscando así una existencia trascendente (Vázquez et al. 2009). En tanto, la segunda clasificación, remite al carácter afectivo del bienestar, donde su presencia se determina a partir de la ocurrencia de afectos positivos y la elusión de aquellos negativos. A su vez, también incluye a la satisfacción vital, que remite a juicios cognitivos de diferentes aspectos de la vida individual (Oyanedel \& Mella, 2014; Diener, 2009b). De acuerdo a esta distinción, el componente afectivo del bienestar hedónico se encuentra determinado principalmente por factores de corto plazo, mientras que la satisfacción con la vida cuenta con mayor estabilidad a lo largo del tiempo.

Considerando esta distinción, el artículo tiene por objeto comparar indicadores de bienestar subjetivo hedónico, en su vertiente cognitiva, entre regiones chilenas, comparando en particular las regiones extremas del país, Magallanes y Arica. Esta comparación permitirá contrastar si el nivel socioeconómico así como procesos psicosociales, como la identidad colectiva, juegan un papel específico en el bienestar. Para ello, se recurre a la encuesta de Caracterización Socioeconómica (CASEN) del año 2011 (Ministerio de Desarrollo Social, 2011), que por primera vez incluyó una medida de satisfacción vital. A través de técnicas estadísticas inferenciales y multivariadas, se encuentra evidencia que permite hipotetizar que en las regiones extremas, pero sobre todo en la región de Magallanes, existe una identidad social regional que contribuye al bienestar de sus habitantes.

El artículo presenta, primero, un breve estado del arte respecto de factores socio demográficos y psicosociales relativos al bienestar, pudiendo así delimitarse aquellas variables a incluir en el análisis y que permitirán comparar las regiones extremas con el resto del país. A continuación, se delimita una caracterización socio demográfica e identitaria de las regiones señaladas, con el objeto de contextualizar los resultados que se obtuvieron. Posteriormente, se expone la metodología del estudio, destacando la fuente de datos, las técnicas de análisis y variables empleadas. Luego, se exponen los resultados principales. Y, finalmente, se discuten las principales implicancias del artículo.

\section{FACTORES SOCIO DEMOGRÁFICOS Y PSICOSOCIALES DEL BIENESTAR}

Estudios previos (Diener et al. 2006; Páez et al. 2004) muestran que el bienestar varía ligeramente según la edad y el sexo. Aunque la satisfacción vital es bastante estable, el bienestar decrece ligeramente con la edad. En relación al género, se constata una ligera asociación entre género masculino y bienestar, aunque muchos estudios no encuentran diferencias entre hombres y mujeres (Diener, 2012; Páez et al. 2004). El bienestar subjetivo se asocia con mayor fuerza a indicadores de nivel socioeconómico, como los ingresos y el nivel educacional (Diener, 2012). También se relacionan al desarrollo económico contextual, ya que las regiones y naciones con mayor índice de desarrollo humano (IDH), más ricas, educadas y con mejores expectativas de vida, muestran mayor bienestar colectivo o medio, siendo la relación entre ingresos más fuerte que a nivel individual (Basabe et al. 2002). El estatus socio-económico se asocia también al bienestar eudaimónico o a los criterios de salud mental de 
auto-aceptación o auto-estima, de control del medio o locus de control, de sentido en la vida $y$ de crecimiento o desarrollo personal, aunque la asociación es moderada entre clase social y autoestima (Páez et al. 2004). El bienestar se asocia a la salud física percibida, siendo esta relación mayor que con indicadores objetivos del tipo de evaluaciones médicas. Hay que destacar que la percepción subjetiva de salud antes mencionada es un predictor específico de la sobrevida, aun controlando el estilo de vida y otros indicadores de riesgo. Esto sugiere que esta percepción subjetiva de salud es un factor predictor importante del bienestar en sí mismo y que debe ser tomado en cuenta (Diener et al. 2006; Páez et al. 2004).

Finalmente, una serie de estudios sugieren que la identidad social o colectiva se asocia al bienestar individual (Haslam et al. 2009). La existencia de una fuerte identidad colectiva, de una identificación intensa con la comunidad, una valoración positiva de esta o auto-estima colectiva, una percepción de capacidad de acción o autoeficacia colectiva elevada, son todos procesos de bienestar social que potencian el bienestar individual (Páez et al. 2011). Este estudio busca justamente contrastar esta hipótesis de forma indirecta, ya que la región de Magallanes se caracteriza por un perfil socioeconómico y de identidad colectiva especifica.

\section{REGIONES DE CHILE E IDENTIDAD COLECTIVA}

La división político-administrativa chilena consagra una estructura basada en 15 regiones, las cuales poseen relativa autonomía respecto de decisiones sobre inversión de recursos públicos. A pesar de diferentes intentos descentralizadores a lo largo de su historia, persiste un alto centralismo (Montecinos, 2005; Mardones, 2006), lo que sumado al relegamiento a un segundo plano a las regiones extremas (Correa, 2013), puede influenciar en la conformación de fuertes identidades regionales.

La región de Magallanes presenta un promedio de ingresos superior al resto del país, con tasas de indigencia, pobreza y desocupación, por debajo del promedio nacional y ocupa el segundo lugar en Índice de desarrollo humano en Chile, con el valor de .778, siendo solo inferior a la Región
Metropolitana (MIDEPLAN \& PNUD, 2006). Por todo esto es posible predecir que tendrá un nivel de satisfacción vital superior al resto de las regiones.

También es una región aislada, con fuerte tradición de inmigración y con una identidad regional muy marcada. Estrada y Avendaño (2008) encontraron que la mayoría de una muestra de estudiantes compartía creencias esencialistas sobre la identidad magallánica, como que ser magallánico es hereditario, es parte de lo más profundo de la identidad y debe ser protegida de otras culturas. Otro estudio con muestras de estudiantes evidenció que la identidad regional magallánica era superior a la media de otras regiones del país, además de ser la identidad regional más fuerte que la nacional, mientras que en las otras regiones de Chile la identificación con la nación era mayor que con la región (Zúñiga \& Asún, 2004, 2010). Asimismo, otra investigación encontró que los atributos que definía a la región eran el aislamiento, la seguridad y la tranquilidad, la belleza ambiental, la cooperación y solidaridad (Zúñiga \& Asún, 2013). A su vez, los propios habitantes de la región presentan tasas que bordean el $90 \%$ respecto de la identificación con la zona, destacando entre sus características principales el ser valientes y sacrificados, poseyendo así un autoconcepto positivo (Molina, 2011). Como explicaciones se ha planteado el origen multicultural por la fuente inmigración, aislamiento geográfico y la debilidad de la presencia del estado nación chileno en esa región (Estrada \& Avendaño, 2008). En todo caso, se puede concluir que en un contexto de aislamiento, de difíciles condiciones ambientales, y de percepción de poco apoyo por parte del gobierno central, la fuerte identificación regional positiva se asociaba a un clima socioemocional positivo de solidaridad (Páez et al. 2011).

Por otro lado, la región del extremo norte - Arica-Parinacota también se caracteriza por el aislamiento, la lejanía geográfica y por una percepción de falta de interés por parte del centro del país. También comparten que la región se caracteriza por la tranquilidad, la seguridad y el multiculturalismo (Reveco-Quiroz \& Zúñiga, 2014). Arica-Parinacota y Magallanes son las dos regiones donde la confianza general en la gente es más alta en Chile, rondando el 50\% (Thayer Correa, 
2011), lo que sugiere que ambas se caracterizan por un clima socioemocional más positivo que la media chilena (que es del $40 \%$ ). Sin embargo, al contrario que Magallanes, Arica tienen indicadores de desarrollo social inferiores, aunque se sitúa en un quinto lugar y su IDH es superior a la media nacional, alcanzando a .775 frente a .773 (MIDEPLAN \& PNUD, 2006) y se representa socialmente como una región en crisis, que no progresa, o con problemas de delincuencia $y$ narcotráfico, que se caracteriza por la emigración. También al contrario que Magallanes, la identidad regional si bien se comparte es menos intensa que la nacional, Arica-Parinacota, se caracterizaría por un fuerte patriotismo, anclado en la narrativa oficial de la Guerra del Pacifico, encarnadas en el Morro de Arica, en el himno de la región y la conmemoración del triunfo militar durante esa guerra, así como en su carácter de zona fronteriza (Reveco-Quiroz \& Zúñiga, 2014).

Por ende, es posible predecir que ambas regiones por su identidad más marcada, así como por situarse por encima de la media nacional en IDH, mostrarán mayor bienestar. Además, Magallanes se caracterizará por un nivel de bienestar mayor, ya que si bien Arica comparte elementos positivos de identidad regional, esta se caracteriza por una cierta devaluación, además que de que posee un mejor desarrollo social. Ahora bien, si la explicación del mayor bienestar es sólo económica o se explica por diferencias etarias, de género o de salud física percibida, esta superioridad desaparecerá al controlar estos factores, considerando que se espera una asociación entre sexo masculino, menor edad y mayores ingresos con bienestar. Finalmente, además de un efecto directo, sobre la base de la evidencia se presupone que la región de mayor desarrollo social y mayor identidad colectiva positiva, por su mejor clima emocional, va a jugar un rol de atenuación de las diferencias entre status (roles de género) y socioeconómicos (de nivel de ingreso).

\section{MÉTODO}

\section{Muestra}

Este estudio compara el bienestar subjetivo en Magallanes y Arica con el resto del país utilizando los datos de la encuesta de
Caracterización Socioeconómica (CASEN) del año 2011 ( $\mathrm{N}=200232)$, efectuada por el Ministerio de Desarrollo Social de Chile. Cabe destacar que la muestra tiene representatividad nacional, regional y comunal, contando además con factores de expansión para el análisis particular de la satisfacción vital.

\section{Instrumentos}

Para el análisis del bienestar subjetivo se utilizó el ítem de satisfacción con la vida de esta encuesta, que es una versión modificada de la escala Cantril: "Considerando todas las cosas, ¿cuán satisfecho está usted con su vida en este momento?" con un rango de respuesta de "completamente insatisfecho" = 1 a 10 = "totalmente satisfecho".

A su vez, se incluyeron las variables de edad, quintiles de ingreso autónomo del hogar y sexo, buscando así identificar diferencias entre los diferentes grupos que se conforman a partir de estos indicadores, respecto de su satisfacción vital por regiones.

\section{Método}

Para el cumplimiento del propósito del estudio se realizaron pruebas $t$-student y Anova de un factor con la variable de satisfacción vital, de acuerdo a los grupos conformados por regiones, sexo, edad y quintiles de ingreso, para determinar de este modo la existencia de diferencias estadísticamente significativas. Asimismo, se avanzó sobre la elaboración de modelos multivariados, en particular regresiones lineales múltiples, con bienestar subjetivo como dependiente.

\section{RESULTADOS}

\section{Regiones y Bienestar}

A continuación, se presentan comparaciones en niveles de satisfacción vital por zonas geográficas extremas (Magallanes y Arica y Parinacota) con el resto del país, incluyendo variables socio demográficas como sexo y edad. En primer lugar, se consideran las primeras zonas en conjunto y, luego, por separado, buscando contrastar la primera hipótesis, que indica que las zonas extremas muestran mayor satisfacción vital en general. 
Tabla 1. Satisfacción vital por zonas extremas en conjunto.

\begin{tabular}{lcc}
\cline { 2 - 3 } & \multicolumn{2}{c}{$\begin{array}{c}\text { Considerando todas las cosas, ¿cuán satisfecho está usted con } \\
\text { su vida en este momento? }\end{array}$} \\
\cline { 2 - 3 } & Media & Desviación típica \\
\hline Arica y & 7.567 & 2.032 \\
Magallanes & 7.166 & 2.186 \\
Resto del país & & \\
\hline
\end{tabular}

Tabla 2. Satisfacción vital por zonas extremas diferenciadas.

\begin{tabular}{ccc}
\cline { 2 - 3 } & \multicolumn{2}{c}{$\begin{array}{c}\text { Considerando todas las cosas, ¿cuán satisfecho está usted } \\
\text { con su vida en este momento? }\end{array}$} \\
\cline { 2 - 3 } & Media & Desviación típica \\
\hline Magallanes & 7.954 & 1.982 \\
Arica y Parinacota & 7.214 & 2.013 \\
Resto del país & 7.166 & 2.186 \\
\hline
\end{tabular}

Tabla 3. Satisfacción vital por zonas extremas en conjunto y sexo.

\begin{tabular}{cccc} 
& & \multicolumn{2}{c}{$\begin{array}{c}\text { Considerando todas las cosas, ¿cuán satisfecho } \\
\text { está usted con su vida en este momento? }\end{array}$} \\
\cline { 3 - 4 } & & Media & Desviación típica \\
\hline Arica y & Hombre & 7.771 & 1.903 \\
Magallanes & Mujer & 7.363 & 2.134 \\
Resto del & Hombre & 7.278 & 2.130 \\
país & Mujer & 7.063 & 2.232 \\
\hline
\end{tabular}

La Tabla 1 muestra que los habitantes de zonas extremas reportan niveles de satisfacción vital significativamente mayores que los del resto del país, $t(269557.814)=99.163, p<0.01$.

A continuación, en la Tabla 2 , se contrasta la idea que la región de mayor desarrollo social y más fuerte identidad social, es decir Magallanes, muestra mayor bienestar.

Existe asociación entre las regiones consideradas y la satisfacción vital $F$ $(2,13393112)=7966.097, p<0.01$. De este modo, es posible afirmar que frente a este indicador, las medias de magallánicos son diferentes de ariqueños y el resto de los habitantes del país. Asimismo, la prueba HSD de Tukey indica que las diferencias entre cada par de grupos son estadísticamente significativas. Es decir, si bien los del extremo norte están ligeramente más satisfechos que el resto del país, lo están menos que los magallánicos. Como prueba de contraste complementaria se calculó una correlación entre el bienestar y las tres regiones diferenciadas, en el que se le asignó un 3 a Magallanes, un 2 Arica y un 1 al resto del país, siguiendo el rango esperado de bienestar. La asociación fue significativa, r (200.236)=.03, $p<.0001$, ratificando lo ya expuesto a partir de las pruebas anteriores.

\section{Regiones, género y bienestar}

La comparación de medias permite constatar si la asociación entre sexo masculino y bienestar se dan en general y en cada región. Además, la comparación entre géneros permite controlar este factor.

En Arica y Magallanes los hombres puntúan más alto en la variable de satisfacción vital que las mujeres, tal como se muestra en la Tabla 3, siendo significativa esta diferencia, $t$ $(254174.729)=51.216, p<0.01$. En el resto del país se reitera este comportamiento, en tanto que los hombres tienen una media más alta, $t$ 
Tabla 4. Satisfacción vital por zonas y sexo.

\begin{tabular}{cccc}
\cline { 3 - 4 } & & \multicolumn{2}{c}{$\begin{array}{c}\text { Considerando todas las cosas, ¿cuán satisfecho } \\
\text { está usted con su vida en este momento? }\end{array}$} \\
\cline { 3 - 4 } Magallanes & Media & Desviación típica \\
\hline Arica y & Mujer & 8.039 & 1.908 \\
Parinacota & Hombre & 7.868 & 2.050 \\
Resto del & Mujer & 7.526 & 1.865 \\
país & Hombre & 6.900 & 2.105 \\
& Mujer & 7.278 & 2.130 \\
\hline
\end{tabular}

$(13112909.22)=178.191, p<0.01$.

A su vez, tal como se muestra en la Tabla 4, los hombres de las zonas extremas puntúan más alto que sus pares del resto del país, $t$ $(135838.363)=91.922, p<0.01$, al igual que las mujeres, $t(133944.887)=49.820, p<0.01$. Es decir, la diferencia entre regiones se mantiene controlando el sexo. Asimismo, la diferencia de género estimada por la diferencia entre medias de hombres y mujeres es mayor en los extremos (.41) que en el resto del país (.22).

En Magallanes los hombres reportan mayores niveles de satisfacción vital que las mujeres, siendo estadísticamente significativa esta diferencia, $t(122322.219)=15.151, p<0.01$. Este mismo patrón se reitera para Arica y Parinacota, donde los hombres obtienen una media más alta, $t(132606.871)=57.819, p<0.01$; y para el resto del país, $t(13112909.22)=178.191, p<0.01$.

A su vez, los hombres poseen medias estadísticamente diferentes en las tres zonas abordadas, $F(2,6407409)=4343.163, p<0.01$, lo que contrastado con la prueba HSD de Tukey indica que los magallánicos están más satisfechos con sus vidas que los ariqueños $y$, estos últimos, puntúan más alto que los habitantes del resto del país.

Las mujeres también poseen medias que difieren significativamente, $F$ $(2,6985700)=4169.309, p<0.01$. Sin embargo, aun cuando entre sí los tres grupos son estadísticamente diferentes, las magallánicas son las que más alto puntúan, seguidas de las habitantes de zonas no extremas y en último lugar se encuentran las ariqueñas.

La diferencia entre regiones extremas diferenciadas se mantiene controlando el sexo. Sin embargo, la diferencia de género es mayor (.63) en el extremo norte que en el resto del país (.22) $y$ es ligeramente menor en Magallanes (.17). Sin embargo, en contra de lo anterior, la correlación entre género (hombres $=2$, mujeres $=1$ ) y bienestar fue de $r=.05$ en el resto de las regiones, de $r=.04$ en Magallanes y de $r=.02$ en Arica. Es decir, si bien se confirma que la diferencia es menor en Magallanes que en el resto, no se confirma que el género se asocia más fuertemente al bienestar en Arica.

Región, edad y bienestar

La comparación de medias permitirá constatar si la asociación entre menor edad y bienestar se dan a nivel país y en cada región. Además, el contraste entre edades permite controlar este factor.

En las zonas de Arica y Magallanes, consideradas en conjunto, tal como se muestra en la Tabla 5, los grupos etarios presentan diferencias significativas respecto de la satisfacción vital, $F$ $(3,257715)=1224.506, \quad p<0.01$. A su vez, la prueba HSD de Tukey indica que cada par de variables se diferencia estadísticamente entre sí, por lo que ordenadas de forma decreciente los jóvenes son quienes tienen mayor puntuación, seguidos del tramo 31 a 45, 46 a 59 y 60 o más años.

En tanto, el resto del país también experimenta diferencias estadísticamente significativas respecto de la variable en estudio, $F$ $(3,13135391)=22655.991, \quad p<0.01$, siendo los grupos disímiles entre sí en su totalidad. De este modo, los más jóvenes tienen mayores niveles de satisfacción, seguidos del grupo que abarca desde 31 a 45 años, los adultos mayores y el tramo de 46 a 59 años.

Asimismo, también es posible constatar que 
Tabla 5. Satisfacción vital por zonas extremas en conjunto y tramos etarios.

\begin{tabular}{cccc} 
& & \multicolumn{2}{c}{$\begin{array}{c}\text { Considerando todas las cosas, ¿cuán satisfecho } \\
\text { está usted con su vida en este momento? }\end{array}$} \\
\cline { 3 - 4 } & 15 a 30 años & 7.854 & Desviación típica \\
\hline Arica y & 31 a 45 años & 7.636 & 1.866 \\
Magallanes & 46 a 59 años & 7.404 & 1.941 \\
& 60 o más años & 7.217 & 2.231 \\
Resto del & 15 a 30 años & 7.376 & 2.071 \\
país & 31 a 45 años & 7.079 & 2.089 \\
& 46 a 59 años & 7.019 & 2.208 \\
& 60 o más años & 7.052 & 2.253 \\
\hline
\end{tabular}

Tabla 6. Satisfacción vital por zonas extremas diferenciadas y tramos etarios.

\begin{tabular}{|c|c|c|c|}
\hline & & \multicolumn{2}{|c|}{$\begin{array}{c}\text { Considerando todas las cosas, ¿cuán } \\
\text { satisfecho está usted con su vida en este } \\
\text { momento? }\end{array}$} \\
\hline & & Media & Desviación típica \\
\hline \multirow{5}{*}{ Magallanes } & 15 a 30 años & 8.164 & 1.852 \\
\hline & 31 a 45 años & 8.038 & 1.904 \\
\hline & 46 a 59 años & 8.133 & 2.004 \\
\hline & 60 o más años & 7.412 & 2.082 \\
\hline & 15 a 30 años & 7.663 & 1.849 \\
\hline \multirow{3}{*}{$\begin{array}{c}\text { Arica y } \\
\text { Parinacota }\end{array}$} & 31 a 45 años & 7.247 & 1.898 \\
\hline & 46 a 59 años & 6.578 & 2.186 \\
\hline & 60 o más años & 6.981 & 2.032 \\
\hline \multirow{4}{*}{$\begin{array}{l}\text { Resto del } \\
\text { país }\end{array}$} & 15 a 30 años & 7.376 & 2.089 \\
\hline & 31 a 45 años & 7.079 & 2.208 \\
\hline & 46 a 59 años & 7.019 & 2.253 \\
\hline & 60 o más años & 7.052 & 2.228 \\
\hline
\end{tabular}

para todos los grupos etarios, las zonas extremas presentan niveles estadísticamente superiores al resto del país, $t \quad(85953.236)=72.590$, $p<0.01, \quad t \quad(65741.286)=70.840, \quad p<0.01$, $t \quad(64984.386)=42.620, \quad p<0.01$ y $t$ $(53200.125)=17.735, \quad p<0.01$. Es decir, las diferencias entre regiones se mantienen controlando la edad.

En la región de Magallanes, tal como se muestra en la Tabla 6, existen diferencias estadísticamente significativas en los niveles de satisfacción vital por tramos etarios, $F$ $(3,122950)=940.090, p<0.01$. De este modo, quienes tienen entre 15 y 30 y 46 y 49 años son los que mayor satisfacción reportan, siendo equivalentes sus puntuaciones. El primer grupo difiere estadísticamente de los pertenecientes al rango que oscila entre 31 a 45 años, pero el segundo no. En tanto, los adultos mayores poseen los valores más bajos en esta escala, diferenciándose de todos los demás grupos.

En Arica y Parinacota también existen diferencias estadísticamente significativas entre los grupos etarios, $F(3,134760)=2005.306$, $p<0.01$. Asimismo, todos los tramos difieren estadísticamente entre sí, por lo que al ordenarlos de acuerdo a sus niveles de satisfacción vital se encuentran: 15 a 30, 31 a 45, 60 o más y 46 a 
59 años.

En el resto del país, se reitera el comportamiento observado en Arica $y$ Parinacota, en la medida que los grupos etarios tienen medias estadísticamente diferentes, $F$ $(3,13135391)=22655.991, p<0.01$. Así, los más jóvenes puntúan más alto, seguidos del tramo 31 a 45 años, adultos mayores y 46 a 59 años.

La asociación lineal, finalmente, entre edad y bienestar se confirma en general, ya que la correlación entre edad y bienestar era de $\mathrm{r}=-.12$ en Magallanes, de $r=.18$ en Arica y de $r=-.06$ en el resto.

\section{Región, ingreso y bienestar}

Finalmente, se compararon los quintiles de ingresos en satisfacción para contrastar la asociación entre ingreso y bienestar. Además, la comparación entre niveles de ingresos similares

Tabla 7. Satisfacción vital por zonas extremas en conjunto y quintiles de ingreso.

\begin{tabular}{cccc} 
& & \multicolumn{2}{c}{$\begin{array}{c}\text { Considerando todas las cosas, ¿cuán satisfecho } \\
\text { está usted con su vida en este momento? }\end{array}$} \\
\cline { 3 - 4 } & I & Media & Desviación típica \\
\hline Arica y & II & 7.882 & 2.267 \\
Magallanes & III & 7.608 & 2.112 \\
& IV & 7.672 & 1.981 \\
& V & 8.300 & 1.821 \\
Resto del país & I & 6.512 & 1.696 \\
& II & 6.877 & 2.247 \\
& III & 7.145 & 2.304 \\
& IV & 7.406 & 2.129 \\
& V & 7.903 & 2.089 \\
\hline
\end{tabular}

Tabla 8. Satisfacción vital por zonas extremas diferenciadas y quintiles de ingreso.

\begin{tabular}{cccc} 
& & \multicolumn{2}{c}{$\begin{array}{c}\text { Considerando todas las cosas, ¿cuán satisfecho } \\
\text { está usted con su vida en este momento? }\end{array}$} \\
\cline { 3 - 4 } Media & & Desviación típica \\
\hline \multirow{3}{*}{ Magallanes } & I & 7,375 & 2,172 \\
& II & 7,795 & 2,089 \\
& III & 7,919 & 2,041 \\
Arica y & IV & 8,119 & 1,731 \\
Parinacota & V & 8,641 & 1,530 \\
& I & 6,256 & 2,230 \\
& II & 6,929 & 2,048 \\
& III & 7,362 & 1,897 \\
Resto del país & IV & 7,304 & 1,810 \\
& V & 7,992 & 1,778 \\
& I & 6,512 & 2,247 \\
& II & 6,877 & 2,304 \\
& III & 7,145 & 2,129 \\
& IV & 7,406 & 2,089 \\
& V & 7,903 & 1,879 \\
\hline
\end{tabular}


pertenecientes a regiones diferentes permite controlar este factor.

En Arica y Magallanes los niveles de satisfacción vital están asociados con los quintiles de ingreso, tal como se muestra en la Tabla 7, F $(4,257492)=3266.695, p<0.01$. Asimismo, esta diferencia se presenta al comparar todos los pares de grupos, por lo que a mayor ingreso, es posible aseverar, que aumenta el bienestar subjetivo. El mismo comportamiento se reitera en el resto del país, $F(4,13117829)=148825.260, p<0.01$.

A su vez, todas las medias por quintiles son superiores en las zonas extremas respecto del resto del país, $t(47834.433)=34.795$, $p<0.01, \quad t \quad(55099.357)=49.702, \quad p<0.01$, $t \quad(61615.304)=56.048, \quad p<0.01, \quad t$ $(53285.618)=34.470, p<0.01$ y $t(51701.412)=$ $51.276, p<0.01$.

De este modo, se confirma la asociación entre ingresos y bienestar, así como las diferencias entre regiones, aun controlando el nivel de ingresos.

En Magallanes existen diferencias estadísticamente significativas por quintil de ingreso, de acuerdo a lo obervado en la Tabla $8, F$ $(4,122949)=1393.539, p<0.01$, siendo disímiles las medias respeto de satisfacción vital entre todos los pares de cruces de quintiles. En Arica y Parinacota también existen diferencias entre los quintiles, $F(4,134537)=2479.838, p<0.01$, siendo las disimilitudes estadísticamente diferentes entre todos los pares de quintiles. A diferencia del caso anterior, donde existía una relación donde a mayor ingreso, mayor bienestar, esto se verifica en cuatro de los cinco grupos. En el resto del país se reitera la asociación entre ingresos y bienestar, existiendo diferencias entre todos los grupos, $F$ $(4,13117829)=148825.260, p<0.01$, y pudiendo ordenarse conforme aumenta el ingreso.

Asimismo, en todos los casos, las medias por quintil son diferentes de acuerdo a la zona de pertenencia, F $(2,2440584)=2024.788$, $p<0.01, \quad F \quad(2,2810126)=1977.077, \quad p<0.01$, $F \quad(2,2908892)=1866.909, \quad p<0.01, \quad F$ $(2,2754900)=1365.322, \quad p<0.01 \quad$ y $F$ $(2,2460813)=1818.031, p<0.01$. En los quintiles del I al V (aunque diferencias significativas se dan en quintiles II y III) las medias son mayores en Magallanes, seguida de Arica y Parinacota y del resto del país. Sin embargo, en los quintiles I, IV y $\mathrm{V}$, el orden de las últimas dos zonas se invierte, siendo el extremo norte el menos satisfecho con su vida.

Es decir, en Magallanes son los niveles de ingresos medio-bajos los que más se benefician del mejor clima social de esa zona. Al contrario, los más pobres y más ricos en el extremo norte muestran menor satisfacción que sus equivalentes del resto del país.

Un análisis de la varianza usando a las tres regiones y a los quintiles de ingreso como variables independientes y al bienestar como variable dependiente, así como al sexo y edad como variables, además de constatar el efecto significativo de todas las variables independientes y covariables, confirmó un efecto de interacción significativo, $\mathrm{F}(8,200294)=97.2, \mathrm{p}<.0001$. Esto muestra que los quintiles operan de forma diferencial según las regiones. La diferencia entre el quintil más alto y el más bajo es de 1,23 en Magallanes, de 1,74 en Arica y de 1,4 en el resto del país. Coherentemente con esto, la correlación del bienestar subjetivo con los ingresos era menor de $r=.20$ en Magallanes, más alta de $r=25$ en Arica y media $r=.21$ en el resto. Esto sugiere que el clima social de Magallanes atenúa las diferencias entre grupos sociales, mientras que estas se potencian en Arica.

Finalmente, se llevó a cabo un análisis de regresión múltiple con el bienestar como variable predicha o dependiente y los ingresos, el sexo, la edad, la salud física percibida y la ponderación de regiones (Magallanes $=3$, Arica $=2$, Resto $=1$ ) como variables independientes o predictores. La correlación bivariada entre salud física y bienestar es de .27, $\mathrm{p}<.0001$. La regresión múltiple fue significativa, F $(5,200294)=302355.502$, $p<.0001$, explicando el $10.2 \%$ de la varianza. Los coeficientes betas fueron todos significativos, $\mathrm{t}<$ que 38, todos $\mathrm{p}<.0001$ y sus coeficientes estandarizados eran en el caso de la salud percibida $\mathrm{B}=.25$, los ingresos de $\mathrm{B}=.17$, de la edad de $\mathrm{B}=.01$, de la región de $\mathrm{B}=.03$ y del sexo de $\mathrm{B}=.01$.

\section{DISCUSIÓN}

Los resultados permiten confirmar la ligera asociación entre menor edad, ser de sexo masculino, tener mayores ingresos y mejor salud percibida con el bienestar. La asociación lineal 
entre edad y bienestar se ratifica a nivel país, ya que la correlación entre edad y bienestar era de $\mathrm{r}=-$ .12 en Magallanes, de $r=-.18$ en Arica y de $r=-.06$ en el resto, más alta que la evaluada por (Diener et al. 2006).

Con respecto al género, se constata una ligera asociación entre género masculino y bienestar de $r=.04$ en Magallanes, de $r=.02$ en Arica y de $r=.05$ en el resto, similar a los estudios internacionales que encuentran una diferencia de $r=.04$. Se puede constatar que las diferencias de bienestar entre edades y entre hombres y mujeres son ligeras y explican menos del $2 \%$ de la varianza (Diener, 2012; Páez et al. 2004).

El bienestar subjetivo, en tanto, se asoció con más fuerza a los ingresos, con correlaciones similares a las generales. La relación media es de $r=.19$ (Diener, 2012) frente a $r=.20$ en Magallanes, $r=25$ en Arica y $r=.21$ en el resto del país. Finalmente, se encontró una asociación entre salud física percibida y bienestar similar a los resultados internacionales y que explicaba cerca el $9 \%$ de la varianza. Además, al controlar esta variable, la asociación entre edad y bienestar cambiaba de signo, sugiriendo que es la relación entre edad y disminución del estado físico lo que afecta el bienestar subjetivo.

Estos resultados reafirman la importancia de controlar estos factores al hacer comparaciones entre grupos sociales. Ahora bien, controlando el sexo, la edad y los ingresos, alrededor de un $1 \%$ de la varianza se explicaba por la pertenencia a las regiones extremas. Aunque la varianza total explicada parece escasa, hay que recordar que la explicada por el sexo y la edad son similares y que las explicadas por los ingresos no superan el $2 \%$. Un $20 \%$ del total de alrededor del $5 \%$ la varianza total explicada en bienestar por los factores sociodemográficos lo es por la mera pertenencia regional.

Los resultados de este estudio aportan evidencia indirecta respecto a la existencia de una identidad regional magallánica positiva, que actúa como un factor de bienestar. El hecho que la región Magallánica muestre una mayor satisfacción que el resto de regiones del país, aun controlando el sexo, la edad y los ingresos, sugiere que hay una ventaja agregada, probablemente de carácter psicosocial, en esta región.
También se confirma que la región extrema de Arica muestra un ligero bienestar superior a la media nacional, en ella las diferencias de género son menos marcadas, aunque son más marcadas las diferencias entre niveles de ingresos-inclusive en el caso de los más pobres y los más ricos pertenecer a esta región socava el bienestar.

Finalmente, los resultados sobre las diferencias entre quintiles más altos y más bajos, que era menor en Magallanes que en las otras regiones, sugiere que el clima social de esta región, atenúa las diferencias entre grupos sociales, mientras que estas se potencian en Arica. Estos resultados son contradictorios con la visión que se tiene de homogeneidad social en Arica (RevecoQueiroz \& Zúñiga, 2014).

Así, es posible concluir que, además de un efecto directo que probablemente provoca un mayor bienestar en Magallanes, se constata un efecto indirecto o de interacción, que sugiere que la fuerte identidad colectiva y clima emocional positivo de esta región amortigua el impacto de las asimetrías de status y económicas.

Este estudio tiene obviamente limitaciones, que es necesario explicar de manera tal de facilitar un adecuado balance de sus resultados. Primero, la más importante es que no hay un indicador de identificación con la región que permita contrastar la idea que la identificación es más alta en las regiones extremas que en el resto y, sobre todo, que permita examinar si la fuerza de la identificación regional se asocia al bienestar. Segundo, se ha colapsado al resto de las regiones del país, que muestran una gran heterogeneidad tanto en indicadores socioeconómicos como psicosociales, como la confianza interpersonal. Tercero, el efecto de la pertenencia regional se estima de forma residual - es la varianza explicada por la ponderación de las regiones controlando los factores sociodemográficos claros. Aun así, este estudio refuerza la importancia de la identidad social, en particular la colectiva regional, para la comprensión del bienestar.

\section{BIBLIOGRAFÍA}

Anger, E. (2010). Subjective well-being. The Journal of SocioEconomics, 39(3), 361-368.

Basabe, N., Páez, D., Valencia, J., González, J. L., Rimé, B. \& 
Diener, E. (2002). Cultural dimensions, socioeconomic development, climate and emotional hedonic level. Cognition and Emotion, 16, 103-125.

Correa, L. (2013). Políticas públicas y gobernabilidad en las zonas extremas de Chile 2010-2012. Estudios de Seguridad y Defensa, 1, 17-43.

Diener, E., Lucas, R., \& Scollon, C. N. (2006). Beyond the hedonic treadmill: Revising the adaptation theory of well-being. American Psychologist, 61, 305-314.

Diener, E. (2009a). Introduction - The Science of Well-Being: Reviews and Theoretical Articles. In E. Diener (Ed.), The Science of Well-Being. The Collected Works of Ed. Diener (pp. 1-10). New York: Springer.

Diener, E. (2009b). Subjective Well-Being. In E. Diener (Ed.), The Science of Well-Being. The Collected Works of Ed. Diener (pp. 11-58). New York: Springer.

Diener, E. (2012). New findings and future directions for subjective well-being research. American Psychologist, 67, 590-597.

Estrada, C. \& Avendaño, S. (2008). ¿Consideran los magallánicos que su cultura posee una esencia única?: Estudio descriptivo de la aplicación de un instrumento de evaluación de esencialismo cultural a una muestra puntarenense. Magallania, 36(2), 155-162.

Haslam, S. A., Jetten, J., Postmes, T., \& Haslam, C. (2009). Social Identity, Health and Well-Being: An Emerging Agenda for Applied Psychology. Applied Psychology: an International Review, 58(1), 1-23.

Mardones, R. (2006). Descentralización y transición en Chile. Revista de Ciencia Política, 26(1), 3-24.

MIDEPLAN \& PNUD (2006). Las trayectorias del Desarrollo Humano en las Comunas de Chile (1994-2003). Santiago: Programa de las Naciones Unidas para el Desarrollo.

Ministerio de Desarrollo Social (2011). Encuesta CASEN: descripción y objetivos, metodología y base de datos. Recuperado el 14 de Noviembre de 2016, de sitio web del Ministerio de Desarrollo Social: http://observatorio. ministeriodesarrollosocial.gob.cl/casen_obj.php

Molina, W. (2011). Identidad regional en Magallanes, sus expresiones simbólicas y territoriales. Magallania, 39(1), 59-60.

Montecinos, E. (2005). Antecedentes sobre la relación histórica centralismo y descentralización en Chile. Revista Venezolana de Gerencia, 10(31), 443-462.

Oyanedel, J. C. \& Mella, C. (2014). La felicidad como vocación o el bienestar como profesión: una Mirada a la economía política del bienestar y la felicidad. In J. C. Oyanedel \& C. Mella (Ed.), Debates sobre el bienestar y la felicidad (pp. 211-223). Santiago: RIL Editores.

Páez, D., Fernandez, I., Ubillos, S. \& Zubieta, E. (2004). Psicología Social, Cultura y Educación. Madrid: McGraw Hill.

Páez, D., Martin Beristain, C., González, J.L. Basabe, N. \& De Rivera, J. (2011). Superando la Violencia Colectiva y Construyendo cultura de paz. Madrid: Fundamentos.

Reveco-Quiroz, P. \& Zúñiga, C. (2014). Significado valorativo y emocional de la pertenencia a las regiones de O'Higgins y Arica Parinacota. Universum, 29(1) ,107-123.

Thayer Correa, L.E. (2011). Descentralización y desarrollo regional en Chile. Polis, 10(30), 267-287.

Vázquez, C., Hervás, G., Rahona, J. J., \& Gómez, D. (2009). Bienestar psicológico y salud: Aportaciones desde la Psicología Positiva. Anuario de Psicología Clínica y de la Salud, 5, 15-28.

Zúñiga, C. \& Asún, R. (2004). Diseño y Validación de una Escala de Identidad Regional. Revista de PsicologíaSocial, 19(1), 35-49.

Zúñiga, C. \& Asún, R. (2010). Identidad social y discriminación intergrupal ¿Una relación inevitable? El caso de las identidades regionales en Chile. Revista de Psicología Social, 25(2), 215-230.

Zúñiga, C. \& Asún, R. (2013). Identidad regional en estudiantes universitarios de Magallanes: una aproximación psicosocial. Magallania, 41(1), 83-98. 
